\title{
THE GEOMETRY OF CLOSED CONFORMAL VECTOR FIELDS ON RIEMANNIAN SPACES
}

\author{
A. CAMINHA \\ To prof. M. Dajczer on occasion of his $60^{\text {th }}$ birthday
}

\begin{abstract}
In this paper we examine different aspects of the geometry of closed conformal vector fields on Riemannian manifolds. We begin by getting obstructions to the existence of closed conformal and nonparallel vector fields on complete manifolds with nonpositive Ricci curvature, thus partially generalizing a theorem of T. K. Pan. Then we explain why it is so difficult to find examples, other than trivial ones, of spaces having at least two closed, conformal and homothetic vector fields. Finally we focus on isometric immersions, proving general Bernstein-type theorems for certain complete, not necessarily cmc, hypersurfaces of Riemannian manifolds furnished with closed conformal vector fields. In particular, we obtain a generalization of theorems J. Jellett and A. Barros and P. Sousa for complete $\mathrm{cmc}$ radial graphs over finitely punctured geodesic spheres of Riemannian space forms.
\end{abstract}

\section{INTRODUCTION}

Conformal vector fields are relevant objects for the geometry of several kinds of spaces. For example, if $I \subset \mathbb{R}$ is an open interval, $f$ is a positive smooth function on $I$ and $F$ is a Riemannian manifold, the warped product $M=I \times{ }_{f} F$ carries the natural closed conformal vector field $\left(f \circ \pi_{I}\right) \partial_{t}$, where $\pi_{I}: M \rightarrow I$ is the canonical projection and $t$ the standard coordinate on $I$; on the other hand, if one fixes a point $p$ in a Riemannian manifold $M$ of constant sectional curvature $c$, let $r$ be the distance function from $p$ on $M$ and $s$ the solution to the ODE $y^{\prime \prime}+c y=0, y(0)=0$, $y^{\prime}(0)=1$, then $\xi=(s \circ r) \nabla r$ is a conformal vector field out of the cut locus of $p$ in $M$, where $\nabla r$ stands for the gradient of $r$ on $M$. These occurrences of conformal vector fields are two that we shall explore in this paper; as for a third one (cf. [5]), let us mention that if $M$ is a pseudoumbilic submanifold of a complex space form $(\overline{\mathbb{M}}, J)$, and $H$ is the mean curvature vector of $M$ on $\overline{\mathbb{M}}$, then $J H$ is a conformal vector field on $M$.

Due to the range of examples above, it is no surprise that closed conformal vector fields have been taken as natural tools in the geometric study of submanifolds and, more particularly, of hypersurfaces. To mention just some recent work related to this paper, A. Barros, A. Brasil Jr. and the author [1] used the natural closed conformal vector field on a Lorentz warped product with 1 -dimensional basis to classify, under appropriate conditions, strongly stable, closed cmc spacelike hypersurfaces of such a space as being either maximal or spacelike slices; H. F. de

2000 Mathematics Subject Classification. Primary 53C42, 53C45; Secondary 53C65.

Key words and phrases. Conformal vector fields; warped products; Jellett's theorem; Bernstein-type theorems.

The author is partially supported by CNPq. 
Lima and the author [3] studied complete cmc vertical graphs in the hyperbolic and steady state spaces, obtaining necessary conditions for their existence under natural growth restrictions on the corresponding height functions; A. Barros and P. Sousa 2] applied the conformal vector field on constant curvature spaces to extend to the sphere a theorem of J. Jellett [7] on cmc closed radial graphs of $\mathbb{R}^{n+1}$.

In this paper we focus on two different aspects of conformal vector fields on Riemannian spaces, namely, obstructions to their existence and the role they play in Bernstein-type results. We begin, in section 2, refining a result of S. T. Yau [12] (cf. Proposition 2.1), which is then applied to the study of closed conformal vector fields on complete, noncompact, simply connected Riemannian manifolds with nonpositive Ricci curvature; according to Proposition 2.3. provided a suitable integrability condition on the vector field is satisfied, we show it has to be both parallel and a direction of vanishing of the Ricci curvature on the manifold. This result partially generalizes a previous theorem of T. K. Pan [10]. Continuing this study, in Lemma 3.1 of section 3 we give a general geometric construction of closed conformal vector fields on certain hypersurfaces of ambient spaces furnished with two closed conformal and homothetic vector fields; this is then applied, in Theorem 3.4. to explain why it is not easy to give examples, out of the trivial ones, of manifolds with many homothetic vector fields.

Bernstein-type results are dealt with in section 4 For ambient spaces of nonnegative Ricci curvature and possessing a parallel and a homothetic nonparallel vector field, Theorem 4.2 establishes a reasonable set of sufficient conditions for a complete, oriented (not necessarily $\mathrm{cmc}$ ) hypersurface of bounded second fundamental form to be totally geodesic and, more particularly, a leaf of the orthogonal foliation determined by the parallel vector field. This result generalizes our previous work in [4. Next, in Theorem 4.3 we consider again an ambient space of nonnegative Ricci curvature, this time with a single homothetic vector field; under the same set of necessary conditions, we establish the umbilicity of a complete, oriented cmc hypersurface of bounded second fundamental form. We specialize this result to $\mathbb{R}^{n+1} \backslash\{0\}=(0,+\infty) \times{ }_{t} \mathbb{S}^{n}$ in Corollary 4.6, thus generalizing of a theorem of J. Jellett [7] for complete cmc radial graphs over a finitely punctured sphere in Euclidean space. Finally, Theorem 4.7 deals with the corresponding situation in ambients of constant sectional curvature, relying this time on the conformal vector field $\xi=(s \circ r) \nabla r$. Using techniques similar to the ones required for our previous results, Corollary 4.8 generalizes the main theorem of [2] for complete cmc radial graphs over a finitely punctured geodesic sphere of the Euclidean sphere and the hyperbolic space.

\section{ObstruCtions on the EXISTENCE OF CONFORMAL FIELDS}

Let $M^{n}, n \geq 2$, be an $n$-dimensional, oriented Riemannian manifold with metric $g=\langle$,$\rangle and Levi-Civita connection \nabla$. We recall that a smooth vector field $\xi$ on $M$ is conformal if

$$
\mathcal{L}_{\xi} g=2 \psi_{\xi} g
$$

for some smooth function $\psi_{\xi}$ on $M$, where $\mathcal{L}_{\xi}$ denotes the Lie derivative in the direction of $\xi$. The function $\psi_{\xi}$ is the conformal factor of $\xi$.

Since $\mathcal{L}_{\xi}(X)=[\xi, X]$ for every $X \in \mathfrak{X}(M)$, it follows from the tensorial character of Lie derivatives that $\xi \in \mathfrak{X}(M)$ is conformal if and only if there exists a smooth 
function $\psi_{\xi}$ on $M$ such that

$$
\left\langle\nabla_{X} \xi, Y\right\rangle+\left\langle X, \nabla_{Y} \xi\right\rangle=2 \psi_{\xi}\langle X, Y\rangle
$$

for all $X, Y \in \mathfrak{X}(M)$. In particular,

$$
\psi_{\xi}=\frac{1}{n} \operatorname{div}_{M} \xi
$$

Therefore, if $M$ is closed, divergence theorem assures that a necessary condition for the existence of such a vector field $\xi$ is that

$$
\int_{M} \psi_{\xi} d M=0
$$

An interesting particular case of a conformal vector field $\xi$ is that in which $\nabla_{X} \xi=\psi_{\xi} X$ for all $X \in \mathfrak{X}(M)$; in this case we say that $\xi$ is closed, an allusion to the fact that its dual $1-$ form $\omega_{\xi}$ is closed. Yet more particularly, a closed and conformal vector field $\xi$ is said to be parallel if $\psi_{\xi}$ vanishes identically, and homothetic if $\psi_{\xi}$ is constant].

It is easy to prove that the conformal character of a vector field is invariant under a conformal change of metric. On the other hand, a closed conformal vector field remains closed if and only if the conformal change has constant conformal factor, in which case the conformal factor of the field does not change.

From now on, unless stated otherwise, we stick to closed conformal fields. If we further assume $M$ to be simply connected, Poincaré's lemma gives $\omega_{\xi}=d f$, for some smooth function $f$ on $M$. Then $\xi=\nabla f$, $\operatorname{Hess}_{M} f=\psi_{\xi}$ Id and $\psi_{\xi}=\frac{1}{n} \Delta f$. Therefore, if $M$ is closed and simply connected, it follows from Green's first identity that

$$
n \int_{M} \psi_{\xi} f d M+\int_{M}|\nabla f|^{2} d M=\frac{1}{2} \int_{M} \Delta\left(f^{2}\right) d M=0 .
$$

In particular, there are no parallel nontrivial vector fields on $M$. A further restriction on the existence of closed conformal vector fields is given by Bochner's formula (making $\xi=\nabla f$ on it)

$$
\begin{aligned}
\frac{1}{2} \Delta\left(|\xi|^{2}\right) & =\operatorname{Ric}_{M}(\xi, \xi)+n\left\langle\xi, \nabla \psi_{\xi}\right\rangle+n \psi_{\xi}^{2} \\
& =\operatorname{Ric}_{M}(\xi, \xi)+n \operatorname{div}_{M}\left(\psi_{\xi} \xi\right)-n(n-1) \psi_{\xi}^{2},
\end{aligned}
$$

where $\operatorname{Ric}_{M}(\cdot, \cdot)$ denotes the Ricci tensor of $M$. Integrating over $M$ gives

$$
\int_{M} n(n-1) \psi_{\xi}^{2} d M=\int_{M} \operatorname{Ric}_{M}(\xi, \xi) d M .
$$

In particular, there are no closed conformal nontrivial vector fields on a closed, simply connected hyperbolic manifold.

The above result was known to Pan in [10, and we now proceed to generalize it to simply connected, complete noncompact Riemannian manifolds. To this end, we begin by quoting a version of Stokes' theorem on an $n$-dimensional, complete noncompact Riemannian manifold $M$, obtained by S. T. Yau in [12]: if $\omega \in \Omega^{n-1}(M)$

\footnotetext{
${ }^{1}$ Here we diverge a little bit from other papers (e.g. 8]), where homothetic means just conformal with constant conformal factor. The reason is economy: to avoid constantly writing closed and homothetic.
} 
is an $n-1$ differential form on $M^{n}$, then there exists a sequence $B_{i}$ of domains on $M$, such that $B_{i} \subset B_{i+1}, M=\cup_{i \geq 1} B_{i}$ and

$$
\lim _{i \rightarrow+\infty} \int_{B_{i}} \omega=0
$$

Suppose $M$ is oriented by the volume element $d M$, and let $\mathcal{L}^{1}(M)$ be the space of Lebesgue integrable functions on $M$. If $\omega=\iota_{X} d M$ is the contraction of $d M$ in the direction of a smooth vector field $X$ on $M$, then one gets the following consequence of Yau's result.

Proposition 2.1. Let $X$ be a smooth vector field on the complete, noncompact, oriented Riemannian manifold $M^{n}$, such that $\operatorname{div}_{M} X$ doesn't change sign on $M$. If $|X| \in \mathcal{L}^{1}(M)$, then $\operatorname{div} X=0$ on $M$.

Proof. Assume, without loss of generality, that $\operatorname{div}_{M} X \geq 0$. If $\left\{e_{1}, \ldots, e_{n}\right\}$ is an orthonormal frame on an open set $U \subset M$, with coframe $\left\{\omega_{1}, \ldots, \omega_{n}\right\}$, then

$$
\iota_{X} d M=\sum_{i=1}^{n}(-1)^{i-1}\left\langle X, e_{i}\right\rangle \omega_{1} \wedge \ldots \wedge \widehat{\omega}_{i} \wedge \ldots \wedge \omega_{n} .
$$

Since the $(n-1)$-forms $\omega_{1} \wedge \ldots \wedge \widehat{\omega}_{i} \wedge \ldots \wedge \omega_{n}$ are orthonormal in $\Omega^{n-1}(M)$, we get

$$
|\omega|^{2}=\sum_{i=1}^{n}\left\langle X, e_{i}\right\rangle^{2}=|X|^{2} .
$$

Then $|\omega| \in \mathcal{L}^{1}(M)$ and $d \omega=d\left(\iota_{X} d M\right)=(\operatorname{div} X) d M$. Letting $B_{i}$ be as in the preceeding discussion, we get

$$
\int_{B_{i}}(\operatorname{div} X) d M=\int_{B_{i}} d \omega \stackrel{i}{\longrightarrow} 0 .
$$

It now follows from $\operatorname{div} X \geq 0$ on $M$ that $\operatorname{div} X=0$ on $M$.

Remark 2.2. With respect to the above proposition, in 12 Yau considered just the case $X=\nabla f$, for some smooth function $f: M \rightarrow \mathbb{R}$. Although the generalization just proved seems to be a quite small one, we will see that it gives greater flexibility in what concerns applications.

We are now able to state and prove the following result. We remark that (2) reduces to $\psi_{\xi} \in \mathcal{L}^{1}(M)$ if $\xi$ is a bounded (hence complete) vector field.

Proposition 2.3. Let $M^{n}$ be an n-dimensional complete, simply connected Riemannian manifold with nonpositive Ricci curvature, and let $\xi$ be a closed conformal vector field on $M$, with conformal factor $\psi_{\xi}$. If

$$
\int_{M}\left|\psi_{\xi} \xi\right| d M<+\infty
$$

then $\xi$ is parallel and the Ricci curvature of $M$ in the direction of $\xi$ vanishes identically.

Proof. It follows from (1) that

$$
\operatorname{div}_{M}\left(n \psi_{\xi} \xi-\frac{1}{2} \nabla\left(|\xi|^{2}\right)\right)=(n-1) \psi_{\xi}^{2}-\operatorname{Ric}_{M}(\xi, \xi) .
$$


Now, an easy computation gives $\nabla\left(|\xi|^{2}\right)=2 \psi_{\xi} \xi$, so that

$$
\operatorname{div}_{M}\left(\psi_{\xi} \xi\right)=\psi_{\xi}^{2}-\frac{1}{n-1} \operatorname{Ric}_{M}(\xi, \xi)
$$

Since $\operatorname{Ric}_{M} \leq 0$, we get $\operatorname{div}_{M}\left(\psi_{\xi} \xi\right) \geq 0$, and it follows from (2) and Proposition 2.1 that $\operatorname{div}_{M}\left(\psi_{\xi} \xi\right)=0$ on $M$. Back on (3), we get $\psi_{\xi}=0$ and $\operatorname{Ric}_{M}(\xi, \xi)=0$, as we wished to prove.

An interesting class of spaces furnished with closed conformal vector fields is given by the following subclass of warped product spaces: let $F^{n-1}$ be an $(n-$ 1)-dimensional Riemannian manifold with metric $(),, I \subset \mathbb{R}$ be an open interval and $f: I \rightarrow \mathbb{R}_{+}^{*}$ be a smooth function, called the warping function. Set $M^{n}=$ $I \times F^{n-1}$ (as a differentiable manifold), and let $\pi_{I}: M \rightarrow I$ and $\pi_{F}: M \rightarrow F$ denote the canonical projections; we equip $M$ with the warped metric $\langle$,$\rangle , given$ by

$$
\langle X, Y\rangle=d \pi_{I}(X) d \pi_{I}(Y)+\left(f \circ \pi_{I}\right)^{2}\left(d \pi_{F}(X), d \pi_{F}(Y)\right),
$$

and denote the resulting Riemannian space by

$$
M^{n}=I \times_{f} F^{n-1} .
$$

If $\partial_{t}$ is the standard unit vector field on $I$, then $\xi=\left(f \circ \pi_{I}\right) \partial_{t}$ is closed conformal, with conformal factor $\psi_{\xi}=f^{\prime} \circ \pi_{I}$ (cf. 9], Proposition 7.35).

Let $F$ be oriented by the volume element $d F$, and let $d M$ denote the corresponding volume element of $M=I \times{ }_{f} F$. Since, for a vector field $X \in \mathfrak{X}(F)$, we have $\langle X, X\rangle=\left(f \circ \pi_{I}\right)^{2}(X, X)$, it easily follows that

$$
d M=\frac{1}{f^{n-1}} d t \wedge d F .
$$

We also observe that if $F$ is complete and $I=\mathbb{R}$, then Lemma 7.40 of 9 gives $M$ complete, regardless of the warping function $f$. Moreover, if $f$ is constant and $F$ has nonpositive Ricci curvature, then Corollary 7.43 of $[9$ asserts that the same is true of $M=\mathbb{R} \times{ }_{f} F$. In what follows we use Proposition 2.3 to obtain a partial converse to this fact.

Corollary 2.4. Let $n \geq 3, F^{n-1}$ be a closed, simply connected Riemannian manifold and $f: \mathbb{R} \rightarrow \mathbb{R}_{+}^{*}$ be a smooth, nondecreasing bounded function. If $M^{n}=$ $\mathbb{R} \times{ }_{f} F^{n-1}$ has nonpositive Ricci curvature, then one of the following happens:

(a) $f(-\infty)=0$.

(b) $f$ is constant and $F$ has nonpositive Ricci curvature.

Proof. Let $\pi: M \rightarrow \mathbb{R}$ denote the canonical projection. We shall assume that $n>3$, the analysis of the case $n=3$ being totally analogous. It follows from the above discussion that

$$
\begin{aligned}
\int_{M}\left|\psi_{\xi} \xi\right| d M & =\int_{M}\left(\left(f^{\prime} f\right) \circ \pi\right) d M=\int_{M}\left(\frac{f^{\prime}}{f^{n-2}} \circ \pi\right) d t d F \\
& =\operatorname{Vol}(F) \int_{-\infty}^{+\infty} \frac{f^{\prime}}{f^{n-2}} d t=\left.\frac{\operatorname{Vol}(F)}{3-n} f^{3-n}\right|_{-\infty} ^{+\infty} .
\end{aligned}
$$

Since $f$ is bounded, nondecreasing and positive, this is finite if and only if $f(-\infty)>$ 0 . If this is the case, then Proposition 2.3 and our previous discussions give $\psi_{\xi}=$ $f^{\prime} \circ \pi \equiv 0$, so that $f$ is constant. Now, Corollary 7.43 of 9 guarantees that $F$ has nonpositive Ricci curvature. 


\section{Spaces With SEVERAL Homothetic VeCtor FieldS}

We now turn to the analysis of isometric immersions in the presence of conformal vector fields. To this end it is convenient to perform a slight change in the notations, so that from this section on we consider $\tilde{M}^{n+1}, n \geq 2$, an oriented, $(n+1)$-dimensional Riemannian manifold with metric $g=\langle$,$\rangle and Levi-Civita$ connection $\tilde{\nabla}$. We also let $\xi$ be a fixed conformal vector field on $\tilde{M}$, with conformal factor $\psi_{\xi}$.

Let us first analyse the case in which $\xi$ is closed and nontrivial. If it has no singularities on an open set of $\tilde{M}$, let $\xi^{\perp}$ be the distribution, on this open set, of vector fields orthogonal to $\xi$. For $X, Y \in \xi^{\perp}$, we have

$$
\langle[X, Y], \xi\rangle=\left\langle\tilde{\nabla}_{X} Y-\tilde{\nabla}_{Y} X, \xi\right\rangle=-\left\langle Y, \tilde{\nabla}_{X} \xi\right\rangle+\left\langle X, \tilde{\nabla}_{Y} \xi\right\rangle=0
$$

so that $\xi^{\perp}$ is integrable by Frobenius' theorem. We let $\Xi$ be a leaf of $\xi^{\perp}$, furnished with the induced metric, and $D$ be its Levi-Civita connection.

If we ask $\tilde{M}$ to be simply connected, then $\xi=\tilde{\nabla} f$ for some smooth function $f$ on $\tilde{M}$. For a smooth curve segment $\gamma:[0,1] \rightarrow \Xi$, we get

$$
\frac{d}{d t}(f \circ \gamma)(t)=\left\langle\tilde{\nabla} f, \gamma^{\prime}\right\rangle(t)=\left\langle\xi, \gamma^{\prime}\right\rangle(t)=0
$$

so that $f$ is constant along $\gamma$, and hence on $\Xi$. Therefore, $\Xi$ is a connected component of a level set of $f$.

Back to a general $\tilde{M}$, for $X \in \mathfrak{X}(\Xi)$ the closedness of $\xi$ gives

$$
X\langle\xi, \xi\rangle=2\left\langle\tilde{\nabla}_{X} \xi, \xi\right\rangle=2\left\langle\psi_{\xi} X, \xi\right\rangle=0,
$$

i.e., $\langle\xi, \xi\rangle$ is constant on $\Xi$. By rescaling the metric of $\tilde{M}$ by a constant factor we can further ask that $\langle\xi, \xi\rangle=1$ on $\Xi$, so that $\xi$ is a global unit normal vector field on $\Xi$. Therefore, if $S_{\Xi}$ denotes the shape operator of $\Xi$ with respect to $\xi$, we get

$$
S_{\Xi}(X)=-\tilde{\nabla}_{X} \xi=\psi_{\xi} X
$$

and hence $\Xi$ is an umbilical hypersurface of $\tilde{M}$ (totally geodesic if $\xi$ is parallel).

With the above notations, we have the following

Lemma 3.1. If $\eta$ is another closed conformal vector field on $\tilde{M}$ and $U=\eta-\langle\eta, \xi\rangle \xi$, then $U$ is closed conformal on $\Xi$, with conformal factor $\psi_{U}=\psi_{\eta}-\psi_{\xi}\langle\eta, \xi\rangle$.

Proof. For $Z \in \mathfrak{X}(\Xi)$, it follows from $\langle Z, \xi\rangle=0$ that

$$
\begin{aligned}
D_{Z} U= & \left(\tilde{\nabla}_{Z} U\right)^{\top}=\tilde{\nabla}_{Z} U-\left\langle\tilde{\nabla}_{Z} U, \xi\right\rangle \xi \\
= & \tilde{\nabla}_{Z}(\eta-\langle\eta, \xi\rangle \xi)-\left\langle\tilde{\nabla}_{Z}(\eta-\langle\eta, \xi\rangle \xi), \xi\right\rangle \xi \\
= & \tilde{\nabla}_{Z} \eta-Z\langle\eta, \xi\rangle \xi-\langle\eta, \xi\rangle \tilde{\nabla}_{Z} \xi-\left\langle\tilde{\nabla}_{Z} \eta, \xi\right\rangle \xi \\
& +Z\langle\eta, \xi\rangle\langle\xi, \xi\rangle \xi+\langle\eta, \xi\rangle\left\langle\tilde{\nabla}_{Z} \xi, \xi\right\rangle \xi \\
= & \tilde{\nabla}_{Z} \eta-\langle\eta, \xi\rangle \tilde{\nabla}_{Z} \xi-\left\langle\tilde{\nabla}_{Z} \eta, \xi\right\rangle \xi \\
= & \psi_{\eta} Z-\langle\eta, \xi\rangle \psi_{\xi} Z-\left\langle\psi_{\eta} Z, \xi\right\rangle \xi \\
= & \left(\psi_{\eta}-\langle\eta, \xi\rangle \psi_{\xi}\right) Z,
\end{aligned}
$$

The above Lemma allows us to give the following important

Examples 3.2. We now show how to geometrically build closed conformal vector fields on $\mathbb{S}^{n}$ and $\mathbb{H}^{n+1}$. 
(a) For $\mathbb{S}^{n}$, let us take $\xi(x)=x$ in $\mathbb{R}^{n+1}$ (which is homothetic) and a parallel $\eta$; constructing $U \in T \mathbb{S}^{n}$ as above, we get $U$ closed conformal in $\mathbb{S}^{n}$.

(b) For $\mathbb{H}^{n}$ we take the hyperquadric model

$$
\mathbb{H}^{n}=\left\{x \in \mathbb{L}^{n+1} ;\langle x, x\rangle=-1\right\},
$$

where $\mathbb{L}^{n+1}$ is the $(n+1)$-dimensional Lorentz space with its usual scalar product $\langle\cdot, \cdot\rangle$, viewed as a semi-Riemannian metric. Letting $\tilde{\nabla}$ be the corresponding Levi-Civita connection, it is straightforward to extend the notion of conformality to this setting. Therefore, if we take $\xi(x)=x$ and a parallel $\eta$, then $\xi$ is homothetic in $\mathbb{L}^{n+1}$; building $U \in T \mathbb{H}^{n}$ from $\xi$ and $\eta$ as above, we get $U$ closed and conformal2.

We need another lemma.

Lemma 3.3. Let $\tilde{M}^{n+1}, n \geq 3$, be a Riemannian manifold furnished with a homothetic vector field $\xi$, and let $\Xi$ be a leaf of $\xi^{\perp}$. If $R_{\tilde{M}}$ stands for the curvature operator of $\tilde{M}$, then

$$
R_{\tilde{M}}(\xi, X) \xi=0, \forall X \in \mathfrak{X}(\Xi) .
$$

Proof. The proof is an easy computation, taking into account that $\psi_{\xi}$ is constant:

$$
\begin{aligned}
R_{\tilde{M}}(\xi, X) \xi & =\tilde{\nabla}_{X} \tilde{\nabla}_{\xi} \xi-\tilde{\nabla}_{\xi} \tilde{\nabla}_{X} \xi+\tilde{\nabla}_{[\xi, X]} \xi \\
& =\tilde{\nabla}_{X}\left(\psi_{\xi} \xi\right)-\tilde{\nabla}_{\xi}\left(\psi_{\xi} X\right)+\psi_{\xi}[\xi, X] \\
& =\psi_{\xi}\left\{\tilde{\nabla}_{X} \xi-\tilde{\nabla}_{\xi} X+[\xi, X]\right\}=0 .
\end{aligned}
$$

For what comes next, recall that the metric $\langle\cdot, \cdot\rangle$ of $\Xi^{n}$ is Einstein (or that $\Xi$ itself is Einstein) if there exists a smooth $\lambda: \Xi \rightarrow \mathbb{R}$ such that

$$
\operatorname{Ric}_{\Xi}(X, Y)=\lambda\langle X, Y\rangle,
$$

for all $X, Y \in \mathfrak{X}(\Xi)$, where $\operatorname{Ric}_{\Xi}(\cdot, \cdot)$ denotes the Ricci tensor of $\Xi$. In particular, the Ricci map of $\Xi$ satisfies

$$
\operatorname{Ric}_{\Xi}(X)=\lambda X, \forall X \in \mathfrak{X}(\Xi) .
$$

Moreover, if $n \geq 3$, it is a standard fact that $\lambda$ is constant on $\Xi$, so that $\Xi^{n}$ has constant Ricci curvature, equal to $\frac{\lambda}{n-1}$.

We now have the following result, which explains why it is not easy to give examples of manifolds with many linearly independent homothetic vector fields.

Theorem 3.4. Let $\tilde{M}^{n+1}, n \geq 3$, be a Riemannian manifold possessing two homothetic vector fields $\xi$ and $\eta$, with $\xi$ being non-parallel, and $\Xi$ be a complete leaf of $\xi^{\perp}$. If $\Xi$ is an Einstein manifold with positive Ricci curvature, and $\xi$ and $\eta$ are linearly independent in at least one point of $\Xi$, then:

(a) $\Xi$ is isometric to an Euclidean $n$-sphere.

(b) There exist $\epsilon>0$ and a neighborhood $\Omega$ of $\Xi$ in $\tilde{M}$ such that $\Omega$ is isometric to the warped product $(-\epsilon, \epsilon) \times_{f} \Xi$, where $f(t)=e^{-\psi_{\xi} t}$.

\footnotetext{
${ }^{2}$ Actually, this procedure gives closed conformal vector fields on all hyperquadrics of $\mathbb{L}^{n+1}$.
} 
Proof. If $U=\eta-\langle\eta, \xi\rangle \xi$, then Lemma 3.1 asserts that $U$ is closed conformal on $M$. Moreover, $U$ is non-homothetic; in fact, again from that lemma, it suffices to prove that $\langle\eta, \xi\rangle$ is non-constant on $M$. By contradiction, suppose it were constant and let $X \in \mathfrak{X}(\Xi)$. Then

$$
\begin{aligned}
0=X\langle\eta, \xi\rangle & =\left\langle\tilde{\nabla}_{X} \eta, \xi\right\rangle+\left\langle\eta, \tilde{\nabla}_{X} \xi\right\rangle \\
& =\psi_{\eta}\langle X, \xi\rangle+\psi_{\xi}\langle\eta, X\rangle \\
& =\psi_{\xi}\langle\eta, X\rangle
\end{aligned}
$$

so that $\langle\eta, X\rangle=0$. But since this is true for all $X$, we get $\eta \in T \Xi^{\perp}$, and hence $\eta$ and $\xi$ are linearly dependent everywhere on $\Xi$, which is a contradiction.

Since $n \geq 3$, the Einstein manifold $\Xi$ has constant Ricci curvature, hence constant and positive from our hypothesis; since it is complete, Bonnet-Myers theorem gives $\Xi$ compact. In particular, $U$ is complete on $\Xi$, and Theorem 3.1 (ii) of [8] implies that $\Xi$ is isometric to an Euclidean $n$-sphere.

Concerning (b), let $\Phi$ be the flow of $\xi$. The compactness of $\Xi$ allows us to choose $\epsilon>0$ such that $\Phi$ is defined in $(-\epsilon, \epsilon) \times \Xi$. For a fixed $p \in \Xi$, let $\alpha:(-\delta, \delta) \rightarrow \Xi$ be a curve such that $\alpha(0)=p$. Since

$$
\left.\left\langle\frac{D}{\partial t} \Phi(t, \alpha(s)), \frac{D}{\partial s} \Phi(t, \alpha(s))\right\rangle\right|_{t=0}=\left\langle\xi(\alpha(s)), \frac{d \alpha}{d s}\right\rangle=0,
$$

we can suppose that $\epsilon$ was so chosen that $\Phi:(-\epsilon, \epsilon) \times \Xi \rightarrow \tilde{M}$ is an embedding.

We now claim that the inner product on the left hand side of (7) is zero for all $|t|<\epsilon$, and not just $t=0$. In fact, setting $\varphi(t, s)=\left\langle\frac{D}{\partial t} \Phi(t, \alpha(s)), \frac{D}{\partial s} \Phi(t, \alpha(s))\right\rangle$, the conformal character of $\xi$ gives

$$
\begin{aligned}
\frac{\partial \varphi}{\partial t}(t, s) & =\frac{d}{d t}\left\langle\frac{D}{\partial t} \Phi(t, \alpha(s)), \frac{D}{\partial s} \Phi(t, \alpha(s))\right\rangle \\
& =\left\langle\frac{D^{2}}{\partial t^{2}} \Phi(t, \alpha(s)), \frac{D}{\partial s} \Phi(t, \alpha(s))\right\rangle+\left\langle\frac{D}{\partial t} \Phi(t, \alpha(s)), \frac{D}{\partial s} \frac{D}{\partial t} \Phi(t, \alpha(s))\right\rangle \\
& =\left\langle\frac{D}{\partial t} \xi(\Phi(t, \alpha(s))), \frac{D}{\partial s} \Phi(t, \alpha(s))\right\rangle+\left\langle\xi(\Phi(t, \alpha(s))), \frac{D}{\partial s} \xi(\Phi(t, \alpha(s)))\right\rangle \\
& =\psi_{\xi}\left\langle\xi(\Phi(t, \alpha(s))), \frac{D}{\partial s} \Phi(t, \alpha(s))\right\rangle+\psi_{\xi}\left\langle\xi(\Phi(t, \alpha(s))), \frac{D}{\partial s} \Phi(t, \alpha(s))\right\rangle \\
& =2 \psi_{\xi} \varphi(t, s),
\end{aligned}
$$

so that $\varphi(t, s)=e^{2 \psi_{\xi} t} \varphi(0, s)=0$. Therefore, the closed conformal character of $\xi$ assures that all images $t \mapsto \Phi(t, \Xi),|t|<\epsilon$, are leaves of $\Xi^{\perp}$, hence totally umbillic in $\tilde{M}$.

On the other hand, since $\frac{D}{\partial t} \xi(\Phi(t, \alpha(s)))=\psi_{\xi} \xi(\Phi(t, \alpha(s)))$, the curves $t \mapsto$ $\Phi(t, \alpha(s))$ are pre-geodesics in $\Omega=\Phi((-\epsilon, \epsilon) \times \Xi)$. Since $n \geq 2$, it follows from (a) that $\Xi$ is simply connected, and the local version of a theorem of Hiepko (see Theorem 1 and Corollary 1 of [11]) assures that the metric of $\tilde{M}$ on $\Omega$ is warped, say $\Omega$ isometric to $(-\epsilon, \epsilon) \times{ }_{f} \Xi$.

Therefore, Lemma 3.3, together with Proposition 7.42 (2) of [9], gives, for $X, Y \in$ $\mathfrak{X}(\Xi)$,

$$
0=R_{\tilde{M}}(\xi, X) Y=\left(f^{\prime \prime}+\psi_{\xi} f^{\prime}\right) \frac{\langle X, Y\rangle}{f} \xi,
$$

and it follows from $f(0)=1$ that $f(t)=e^{-\psi_{\xi} t}$. 
A slight variant of the proof of item (a) of the above Theorem shows that we can equally treat the case of more homothetic vector fields, thus obtaining the following version.

Theorem 3.5. Let $n, k \in \mathbb{N}$ be given, with $n \geq 2$, and $\tilde{M}^{n+k}$ be a Riemannian manifold possessing $k+1$ homothetic vector fields $\xi_{1}, \ldots, \xi_{k}, \eta$, with at least one of the $\xi_{i}$ being non-parallel, and $\Xi$ be a complete leaf of $\left\langle\xi_{1}, \ldots, \xi_{k}\right\rangle^{\perp}$. If $\Xi$ is an Einstein manifold with positive Ricci curvature, and $\left\{\xi_{1}, \ldots, \xi_{k}, \eta\right\}$ is linearly independent in at least one point of $\Xi$, then $\Xi$ is isometric to an Euclidean $n$-sphere.

\section{Bernstein-type theOREMS}

We continue to employ the notations above. From now on, we let $x: M^{n} \rightarrow$ $\tilde{M}^{n+1}$ be a connected, complete, oriented hypersurface transversal to $\xi$ at every point, $N$ be a unit normal vector field which orients $M$, and $A$ and $H$ respectively the second fundamental form and the mean curvature of $M$ with respect to $N$.

If $f_{\xi}: M \rightarrow \mathbb{R}$ is given by $f_{\xi}=\langle\xi, N\rangle$, the transversality condition above, together with the connectedness of $M$, give that $f_{\xi}$ is either positive or negative on $M$. On the other hand, standard computations (cf. [1]) give

$$
\nabla f_{\xi}=-A\left(\xi^{\top}\right)
$$

and

$$
\Delta f_{\xi}=-n \xi^{\top}(H)-\left(\operatorname{Ric}_{\tilde{M}}(N, N)+|A|^{2}\right) f_{\xi}-n\left\{H \psi_{\xi}+N\left(\psi_{\xi}\right)\right\}
$$

where ()$^{\top}$ stands for orthogonal projections onto $M$.

If $\eta$ is another closed conformal vector field on $\tilde{M}$ and $g: M \rightarrow \mathbb{R}$ is given by $g=\langle\xi, \eta\rangle$, then more standard computations give

$$
\nabla g=\psi_{\xi} \eta^{\top}+\psi_{\eta} \xi^{\top}
$$

The computation of $\Delta g$ is a bit more involving and, as far as we know, not found in the literature, so we present it for the sake of completeness.

Lemma 4.1. Under the above notations, we have

$$
\Delta g=\eta^{\top}\left(\psi_{\xi}\right)+\xi^{\top}\left(\psi_{\eta}\right)+n H\left(\psi_{\xi} f_{\eta}+\psi_{\eta} f_{\xi}\right)+2 n \psi_{\xi} \psi_{\eta}
$$

Proof. Let $\nabla$ be the Levi-Civita connection of $M$. For fixed $p \in M$, choose an orthonormal frame field $\left\{e_{j}\right\}$ on a neighborhood of $p$ in $M$, such that $\left(\nabla_{e_{i}} e_{j}\right)(p)=0$ 
for all $i, j$. Then, at $p$ we have

$$
\begin{aligned}
\Delta g= & \sum_{j} e_{j}\left(e_{j}(g)\right)=\sum_{j} e_{j}\left\langle\psi_{\xi} e_{j}, \eta\right\rangle+\sum_{j} e_{j}\left\langle\xi, \psi_{\eta} e_{j}\right\rangle \\
= & \sum_{j}\left\{\left\langle e_{j}\left(\psi_{\xi}\right) e_{j}, \eta\right\rangle+\psi_{\xi} e_{j}\left\langle e_{j}, \eta\right\rangle\right\}+\sum_{j}\left\{\left\langle\xi, e_{j}\left(\psi_{\eta}\right) e_{j}\right\rangle+\psi_{\eta} e_{j}\left\langle\xi, e_{j}\right\rangle\right\} \\
= & \left\langle\tilde{\nabla} \psi_{\xi}-N\left(\psi_{\xi}\right) N, \eta\right\rangle+\sum_{j}\left\{\psi_{\xi}\left\langle\tilde{\nabla}_{e_{j}} e_{j}, \eta\right\rangle+\psi_{\xi}\left\langle e_{j}, \tilde{\nabla}_{e_{j}} \eta\right\rangle\right\} \\
& +\left\langle\xi, \tilde{\nabla} \psi_{\eta}-N\left(\psi_{\eta}\right) N\right\rangle+\sum_{j}\left\{\psi_{\eta}\left\langle\tilde{\nabla}_{e_{j}} \xi, e_{j}\right\rangle+\psi_{\eta}\left\langle\xi, \tilde{\nabla}_{e_{j}} e_{j}\right\rangle\right\} \\
= & \eta\left(\psi_{\xi}\right)-N\left(\psi_{\xi}\right) f_{\eta}+\sum_{j}\left\{\psi_{\xi}\left\langle A e_{j}, e_{j}\right\rangle f_{\eta}+\psi_{\xi}\left\langle e_{j}, \psi_{\eta} e_{j}\right\rangle\right\} \\
& +\xi\left(\psi_{\eta}\right)-N\left(\psi_{\eta}\right) f_{\xi}+\sum_{j}\left\{\psi_{\eta}\left\langle\psi_{\xi} e_{j}, e_{j}\right\rangle+\psi_{\eta} f_{\xi}\left\langle e_{j}, A e_{j}\right\rangle\right\} \\
= & \left(\eta-f_{\eta} N\right)\left(\psi_{\xi}\right)+\sum_{j}\left\{\psi_{\xi}\left\langle A e_{j}, e_{j}\right\rangle f_{\eta}+\psi_{\xi}\left\langle e_{j}, \psi_{\eta} e_{j}\right\rangle\right\} \\
& +\left(\xi-f_{\xi} N\right)\left(\psi_{\eta}\right)+\sum_{j}\left\{\psi_{\eta}\left\langle\psi_{\xi} e_{j}, e_{j}\right\rangle+\psi_{\eta} f_{\xi}\left\langle e_{j}, A e_{j}\right\rangle\right\} \\
= & \eta^{\top}\left(\psi_{\xi}\right)+\xi^{\top}\left(\psi_{\eta}\right)+n H\left(\psi_{\xi} f_{\eta}+\psi_{\eta} f_{\xi}\right)+2 n \psi_{\xi} \psi_{\eta} .
\end{aligned}
$$

As a consequence of the above computations and Proposition 2.1, one has the following Bernstein-type general theorems for hypersurfaces, the first of which not requiring constant mean curvature.

Theorem 4.2. Let $\tilde{M}$ have nonnegative Ricci curvature, $\xi$ and $\eta$ be respectively a parallel and a homothetic nonparallel vector field on $\tilde{M}^{n+1}$, and $x: M^{n} \rightarrow \tilde{M}^{n+1}$ be as above. If $|A|$ is bounded, $\left|\xi^{\top}\right|$ is integrable and $H$ doesn't change sign on $M$, then:

(a) $M$ is totally geodesic and the Ricci curvature of $\tilde{M}$ in the direction of $N$ vanishes identically.

(b) If $M$ is noncompact and $\operatorname{Ric}_{M}$ is also nonnegative, then $x(M)$ is contained in a leaf of $\xi^{\perp}$.

Proof. Since $\xi$ is parallel and $\eta$ is homothetic and nonparallel, it follows from (8), (10), (91) and (11) that $\nabla f_{\xi}=-A\left(\xi^{\top}\right), \nabla g=\psi_{\eta} \xi^{\top}$,

$$
\Delta f_{\xi}=-n \xi^{\top}(H)-\left(\operatorname{Ric}_{\tilde{M}}(N, N)+|A|^{2}\right) f_{\xi},
$$

and

$$
\Delta g=n H \psi_{\eta} f_{\xi},
$$

with $\psi_{\eta}$ constant and nonzero. Therefore, the hypothesis $\left|\xi^{\top}\right| \in \mathcal{L}^{1}(M)$ guarantees that $|\nabla g| \in \mathcal{L}^{1}(M)$, and the hypothesis on $H$, together with the fact that $\left|f_{\xi}\right|>0$ on $M$ (which is in turn due to the transversality of $M$ and $\xi$ ), assures that $\Delta g$ is either nonnegative or nonpositive on $M$. Therefore, Corollary 1 of [12] gives $\Delta g=0$ on $M$, and hence $H=0$ on $M$.

We now look at (12), which resumes to

$$
\Delta f_{\xi}=-\left(\operatorname{Ric}_{\tilde{M}}(N, N)+|A|^{2}\right) f_{\xi},
$$


and hence doesn't change sign on $M$ too. We also note that the boundedness of $|A|$ on $M$ gives

$$
\left|\nabla f_{\xi}\right| \leq|A|\left|\xi^{\top}\right| \in \mathcal{L}^{1}(M)
$$

As before, these facts give $\Delta f_{\xi}=0$ on $M$, so that

$$
\operatorname{Ric}_{\tilde{M}}(N, N)+|A|^{2}=0
$$

on $M$. Since $\operatorname{Ric}_{\tilde{M}}(N, N) \geq 0$, we then $\operatorname{get}_{\operatorname{Ric}_{\tilde{M}}}(N, N)=0$ and $A=0$ on $M$, i.e., $M$ is totally geodesic. This finishes the proof of $(a)$.

Concerning (b), $A=0$ on $M$ gives $\nabla f_{\xi}=0$ on $M$, so that $f_{\xi}=\langle\xi, N\rangle$ is constant on $M$ and nonzero, due to the transversality assumption. However, $|\xi|^{2}$ is constant on $\tilde{M}$ (since $\xi$ is parallel) and

$$
\left|\xi^{\top}\right|^{2}=|\xi|^{2}-\langle\xi, N\rangle^{2}
$$

so that $\left|\xi^{\top}\right|$ is also constant on $M$. Therefore,

$$
+\infty>\int_{M}\left|\xi^{\top}\right| d M=\left|\xi^{\top}\right| \operatorname{Vol}(M)
$$

But since $M$ is noncompact and has nonnegative Ricci curvature, another theorem of Yau (Theorem 7 of [12]) gives $\operatorname{Vol}(M)=+\infty$, and hence the only possibility is $\left|\xi^{\top}\right|=0$. Therefore, Cauchy-Schwarz inequality gives that $\xi$ is parallel to $N$, and $x(M)$ is contained in a leaf of $\xi^{\perp}$.

We remark that the above result generalizes one of the main results of [4].

Theorem 4.3. Let $\tilde{M}$ have nonnegative Ricci curvature, $\xi$ be a homothetic vector field on $\tilde{M}^{n+1}$, and $x: M^{n} \rightarrow \tilde{M}^{n+1}$ be as before. If $|A|$ is bounded, $\left|\xi^{\top}\right|$ is integrable and $H$ is constant on $M$, then $M$ is totally umbilical and the Ricci curvature of $\tilde{M}$ in the direction of $N$ vanishes identically.

Proof. Since $H$ is constant on $M$ and $\psi_{\xi}$ is constant on $\tilde{M}$, (9) reduces to

$$
\Delta f_{\xi}=-\left(\operatorname{Ric}_{\tilde{M}}(N, N)+|A|^{2}\right) f_{\xi}-n H \psi_{\xi} .
$$

A straightforward computation now gives

$$
\operatorname{div}_{M}\left(\xi^{\top}\right)=n \psi_{\xi}+n H f_{\xi},
$$

so that

$$
\begin{aligned}
\operatorname{div}_{M}\left(\nabla f_{\xi}+H \xi^{\top}\right) & =\Delta f_{\xi}+n H \psi_{\xi}+n H^{2} f_{\xi} \\
& =-\left(\operatorname{Ric}_{\tilde{M}}(N, N)+|A|^{2}-n H^{2}\right) f_{\xi} .
\end{aligned}
$$

Since $\xi$ is transversal to $M, \operatorname{Ric}_{\tilde{M}}(N, N) \geq 0$ and $|A|^{2} \geq n H^{2}$ by Cauchy-Schwarz inequality (with equality if and only if $M$ is totally umbilical), this last expression does not change sign on $M$. Now observe that

$$
\left|\nabla f_{\xi}+H \xi^{\top}\right|=\left|-A \xi^{\top}+H \xi^{\top}\right| \leq(|A|+H)\left|\xi^{\top}\right| \in \mathcal{L}^{1}(M),
$$

so that Proposition 2.3 gives $\operatorname{div}_{M}\left(\nabla f_{\xi}+H \xi^{\top}\right)=0$ on $M$. Back to (14), we then get $\operatorname{Ric}_{\tilde{M}}(N, N)=0$ and $|A|^{2}-n H^{2}=0$, and we already mentioned that this last condition implies $M$ to be totally umbilical.

If $\tilde{M}=I \times{ }_{t} F^{n}$, then Corollary 7.36 and Corollary 7.43 (1) of [9] respectively guarantee that $\Xi_{t_{0}}=\left\{t_{0}\right\} \times F^{n}$ is totally umbilic in $\tilde{M}$ and the Ricci curvature of $\tilde{M}$ in the direction of $\partial_{t}$ indeed vanishes. Therefore, the previous result yields the following corollary on warped products. 
Corollary 4.4. Let $I \subset \mathbb{R}$ be an open interval, $F$ be an $n$-dimensional, complete oriented Riemannian manifold having nonnegative Ricci curvature, $\tilde{M}=I \times_{t} F^{n}$ and $x: M^{n} \rightarrow \tilde{M}^{n+1}$ be as before. If $|A|$ is bounded, $\left|\xi^{\top}\right|$ is integrable and $H$ is constant on $M$, then $M$ is totally umbilical and the Ricci curvature of $\tilde{M}$ in the direction of $N$ vanishes identically. In particular, if $F$ is closed and has positive Ricci curvature everywhere, then $x(M) \subset\left\{t_{0}\right\} \times F$, for some $t_{0} \in I$.

Proof. The first part follows from the theorem. To the second one, if $F$ is closed and has positive Ricci curvature everywhere, then, according to the previous result, the only possible direction for $N$ is that of $\xi=t \partial_{t}$. But if $N$ is parallel to $\partial_{t}$, it is easy to see that $x(M)$ cannot jump from one leaf $\left\{t_{0}\right\} \times F$ to another (remember that $M$ is connected!).

Remark 4.5. Concerning the above corollary, we point out that

$$
\left|\xi^{\top}\right|=|t| \sqrt{1-\left\langle N, \partial_{t}\right\rangle^{2}} .
$$

Therefore, the condition $\left|\xi^{\top}\right| \in \mathcal{L}^{1}(M)$ intuitively amounts to $N$ becoming almost parallel to $\partial_{t}$ as we go to infinity on $M$.

As a special case of the previous corollary, we get a generalization of a theorem of J. Jellett [7] to complete cmc radial graphs over a finitely punctured sphere in Euclidean space.

Corollary 4.6. Let $x: M^{n} \rightarrow \mathbb{R}^{n+1}$ be an embedding, such that $x(M)$ is a complete radial graph over the standard $n$-sphere $\mathbb{S}^{n}$ minus $k \geq 0$ points. If $|A|$ is bounded, $H$ is constant and $p \mapsto\left|x(p)^{\top}\right|$ is integrable on $M$, then $k=0$ and $x(M)$ is a sphere.

We now turn to the case of $\xi$ conformal but not necessarily closed, so that the orthogonal distribution $\xi^{\perp}$ is not necessarily integrable. Since $\psi_{\xi}$ is not necessarily constant, we are obliged to search for an adequate $\xi$.

To this end, let $\tilde{M}$ have constant sectional curvature, say $c$. Following A. Barros and P. Sousa in 2], fix $p \in \tilde{M}$ and let $r: \tilde{M} \rightarrow[0,+\infty)$ be the distance function from $p$ on $\tilde{M}$. If $s: \mathbb{R} \rightarrow \mathbb{R}$ is the solution to the ODE $y^{\prime \prime}+c y=0, y(0)=0$, $y^{\prime}(0)=1$, then

$$
\xi=(s \circ r) \tilde{\nabla} r
$$

is conformal, with conformal factor $\psi_{\xi}=s^{\prime} \circ r$. Therefore,

$$
\begin{aligned}
N\left(\psi_{\xi}\right) & =\left\langle N, \tilde{\nabla} \psi_{\xi}\right\rangle=\left\langle N, \tilde{\nabla}\left(s^{\prime}(r)\right)\right\rangle=\left\langle N, s^{\prime \prime}(r) \tilde{\nabla} r\right\rangle \\
& =-\langle N, c s(r) \tilde{\nabla} r\rangle=-c\langle N, \xi\rangle=-c f_{\xi} .
\end{aligned}
$$

We now observe that formula (9) remains valid for $\xi$ conformal but not necessarily closed (cf. [1]), and hence (recall that $\operatorname{Ric}_{\tilde{M}}(N, N)=c n$ )

$$
\Delta f_{\xi}+n H \psi_{\xi}=-|A|^{2} f_{\xi} .
$$

On the other hand, it is easy to check that (13) also remains valid for $\xi$ conformal but not necessarily closed, and it follows as in the proof of theorem 4.3 that

$$
\operatorname{div}_{M}\left(\nabla f_{\xi}+H \xi^{\top}\right)=-\left(|A|^{2}-n H^{2}\right) f_{\xi}
$$

Taking pieces together we get the following generalization of the main theorems in [2]: 
Theorem 4.7. Let $\tilde{M}^{n+1}$ have constant sectional curvature and $x: M^{n} \rightarrow \tilde{M}^{n+1}$ be as before and such that $x(M) \cap \operatorname{Cut}(p)=\emptyset$ for some $p \in \tilde{M}$. If $|A|$ is bounded, $\left|\xi^{\top}\right|$ is integrable and $H$ is constant on $M$, then $M$ is totally umbilical.

Proof. Fix $q \in M$ and let $\left\{e_{j}\right\}$ be an orthonormal frame field on a neighborhood of $q$ in $M$. For our particular choice of $\xi$, equation (3.1) of [2] gives

$$
\begin{aligned}
e_{j}\langle\xi, N\rangle= & \left\langle\tilde{\nabla}_{e_{j}} \xi, N\right\rangle+\left\langle\tilde{\xi}, \nabla_{e_{j}} N\right\rangle \\
= & \left\langle\tilde{\nabla}_{e_{j}}((s \circ r) \tilde{\nabla} r), N\right\rangle-\left\langle\xi, A e_{j}\right\rangle \\
= & (s \circ r)\left\langle\tilde{\nabla} e_{j} \tilde{\nabla} r, N\right\rangle+\left(s^{\prime} \circ r\right) e_{j}(r)\langle\tilde{\nabla} r, N\rangle-\left\langle A\left(\xi^{\top}\right), e_{j}\right\rangle \\
= & (s \circ r) \cdot \frac{s^{\prime} \circ r}{s \circ r}\left\{\left\langle e_{j}, N\right\rangle-\left\langle e_{j}, \tilde{\nabla} r\right\rangle\langle N, \tilde{\nabla} r\rangle\right\} \\
& +\left(s^{\prime} \circ r\right)\left\langle\tilde{\nabla} r, e_{j}\right\rangle\langle\tilde{\nabla} r, N\rangle-\left\langle A\left(\xi^{\top}\right), e_{j}\right\rangle \\
= & (s \circ r) \cdot \frac{s^{\prime} \circ r}{s \circ r}\left\{\left\langle e_{j}, N\right\rangle-\left\langle e_{j}, \tilde{\nabla} r\right\rangle\langle N, \tilde{\nabla} r\rangle\right\} \\
& +\left(s^{\prime} \circ r\right)\left\langle\tilde{\nabla} r, e_{j}\right\rangle\langle\tilde{\nabla} r, N\rangle-\left\langle A\left(\xi^{\top}\right), e_{j}\right\rangle \\
= & -\left\langle A\left(\xi^{\top}\right), e_{j}\right\rangle .
\end{aligned}
$$

This way, we still have

$$
\nabla f_{\xi}=-\sum_{j}\left\langle A\left(\xi^{\top}\right), e_{j}\right\rangle e_{j}=-A\left(\xi^{\top}\right)
$$

We now just have to follow the steps in the proof of Theorem 4.3, using (15) this time and recalling that $M$ is transversal to $\xi$ by assumption.

The above result extends the main theorem of [2] in two directions, for it covers the compact (closed) case in $\mathbb{H}^{n+1}$ and the complete noncompact case in both $\mathbb{S}^{n+1}$ and $\mathbb{H}^{n+1}$. We explicitly state this extension for the hyperbolic space as the following

Corollary 4.8. Let $x: M^{n} \rightarrow \mathbb{H}^{n+1}$ be an embedding, such that $x(M)$ is a complete radial graph over a geodesic sphere minus $k \geq 0$ points. If $|A|$ is bounded, $H$ is constant and $p \mapsto\left|x(p)^{\top}\right|$ is integrable on $M$, then $k=0$ and $x(M)$ is a geodesic sphere.

\section{REFERENCES}

[1] A. Barros, A. Brasil and A. Caminha. Stability of spacelike hypersurfaces in foliated spacetimes. Diff. Geom. and its Applications 26 (2008) 357-365.

[2] A. Barros and P. Sousa. An Extension of Jellett's Theorem. Bull. Sci. Math., 133 (2008), 190-197.

[3] A. Caminha and H. F. de Lima. Complete vertical graphs with constant mean curvature in semi-Riemannian warped products. Bull. of the Belgian Math. Soc. - Simon Stevin, 16 (2009), 91-105.

[4] A. Caminha, P. Sousa and F. Camargo. Codimension One Foliations of Space Forms. Preprint (2009).

[5] I. Castro, C. Montealegre and F. Urbano. Closed conformal vector fields and Lagrangian submanifolds in complex space forms. Pacific J. of Math., 199 (2001), 269-302.

[6] M. do Carmo. Riemannian Geometry. Boston, Birkhauser (1992).

[7] J. Jellett. La surface dont la courbure moyenne est constant. J. Math. Pures et Appl. 18 (1853) 163-167.

[8] W. Kühnel and H-B Hademacher. Conformal Vector Fields on Pseudo-Riemannian Spaces. Diff. Geom. and its Appl. 7 (1997) 237-250. 
[9] B. O'Neill. Semi-Riemannian Geometry with Applications to Relativity, London, Academic Press (1983).

[10] T. K. Pan. Conformal Vector Fields in Compact Riemannian Manifolds. Proc. of the Amer. Math. Soc. 14 (1963) 653-657.

[11] R. Ponge and H. Reckziegel. Twisted Products in Pseudo-Riemannian Geometry. Geometriae Dedicata 48 (1993), 15-25.

[12] S. T. Yau. Some Function-Theoretic Properties of Complete Riemannian Manifolds and their Applications to Geometry. Indiana Univ. Math. J. 25 (1976), 659-670.

Departamento de Matemática, Universidade Federal do Ceará, Fortaleza, Ceará, BRAZIL. 60455-760

E-mail address: antonio.caminha@gmail.com 